\title{
Effect of Liquid Extract of Pear Avocado Leaf (Persea americana) on Plasma Levels of Aminotransferases, Cholesterol and Total Bile Acids in Hypertensive Patients
}

\author{
Mathew Folaranmi Olaniyan
}

Achievers University, Owo, Ondo State, Nigeria

\begin{abstract}
Raw liquid extract of Avocado pear leaf is of medicinal importance in the treatment of hypertension. In Oke-Ogun area of Oyo state, this extract is being used as an alternative therapy in the treatment of hypertension in human subjects. This work was designed to evaluate the Effect of Liquid Extract of Pear Avocado Leaf used in the Traditional Treatment of Hypertension on the Plasma Levels of Aminotransferases, Total Bile Acids, Total and Low Density Lipoprotein Cholesterol. Fifty (50) anicteric newly diagnosed hypertensive patients with an abnormal increase in plasma cholesterol and blood pressure aged 45 and above that have not been treated with any hypertensive medication but have decided to be treated traditionally using avocado pear leaf extract were recruited from the traditional homes in ATISBO, Saki-East and Saki-West Local government areas of Oke-Ogun - the Northern part of Oyo state-Nigeria. Five (5) milliliter of venous blood was obtained from each subjects after an overnight fasting before and after the administration of the liquid extract for the extraction of plasma. Plasma Alanine amino-transferase(ALT), Aspartate amino-transferase (AST), Total Bile Acids(TBA), Low Density Lipoprotein(LDL) and Total Cholesterol were analysed in the samples of the patients biochemically by spectrophotometry including autoanalysis. The result obtained showed a significantly higher mean value of plasma Total and Low Density Lipoprotein Cholesterol in the subjects before the administration of the liquid extract of avocado pear leaf than the values obtained post-administration with $\mathrm{p}<0.01$. However there was a significantly lower mean value of plasma ALT and AST in the subjects before the administration of the liquid extract of avocado pear leaf than the values obtained after the administration with $\mathrm{p}<0.01$. There was a non-statistically higher plasma value of Total Bile Acids in the subjects before the administration of the raw liquid extract of avocado pear leaf than the value obtained in the subjects after the administration with $p>0.01$. The findings of this study revealed a significant alteration in the plasma level of ALT, AST, LDL and Total Cholesterol in the subjects after the administration of the liquid extrac of avocado pear leaft. The evaluation of these biochemical parameters in hypertensive patients undergoing traditional therapy using the extract is therefore recommended.
\end{abstract}

Keywords Aminotransferases, Hypertension, Liquid Extract, Pear Avocado Leaf, Total/LDL Cholesterol, Total Bile Acids and Traditional Treatment

\section{Introduction}

The medicinal effect of leaves of avocado pear (Persea americana) for the treatment of hypertension has been reported by [1] and [2] in Nigeria and [3] in Ghana. Leaves of avocado pear are cut into pieces, dried and made into tea, for the management of hypertension. Odugbemi [4] has also documented the efficacy of the cotyledons of avocado pear seed for the treatment of hypertension. The cotyledons are cut into pieces, dried and grinded into powder. A dessert spoonful in $200 \mathrm{ml}$ hot water taken after meals gives relieve for the ailment [4]. According to Odugbemi [4] silver bush

* Corresponding author:

olaniyanmat@yahoo.com (Mathew Folaranmi Olaniyan)

Published online at http://journal.sapub.org/ajmms

Copyright (C) 2014 Scientific \& Academic Publishing. All Rights Reserved
(P. pellucida) is also very effective in the treatment of hypertension. This is also in line with earlier reports by Ayitey-Smith [3] in Ghana, and Anslem [1] in Nigeria. Phytochemical analysis of pear leaf revealed Tannins, Saponins, Cardiacglycosides, Alkaloids and Inulin [5].

Cholesterol is required to build and maintain membranes; it modulates membrane fluidity over the range of physiological temperatures. Animal fats are complex mixtures of triglycerides, with lesser amounts of phospholipids and cholesterol. As a consequence, all foods containing animal fat contain cholesterol to varying extents [6]. Major dietary sources of cholesterol include cheese, egg yolks, beef, pork, poultry, fish, and shrimp. Human breast milk also contains significant quantities of cholesterol. Cholesterol is susceptible to oxidation and easily forms oxygenated derivatives known as oxysterols, Cholesterol is oxidized by the liver into a variety of Bile acids [7]. 
Abnormally low levels of cholesterol are termed hypocholesterolemia. Research into the causes of this state is relatively limited, but some studies suggested a link with depression, cancer, and cerebral hemorrhage. In general, the low cholesterol levels seem to be a consequence, rather than a cause, of an underlying illness, contrary to elevated cholesterol level which is associated with heart disease [8].

Bile acids are synthesized in the liver as a breakdown product of cholesterol and secreted into the gall bladder. They are released into the small intestine where they solubilize dietary lipids such as cholesterol, aiding their absorption. Bile acids are reabsorbed from the portal blood by hepatocyte extraction and re-excreted into bile, passing through the enterohepatic circulation several times before final excretion. The measurement of bile acids in serum is a sensitive indicator of liver function [7].

Low-density lipoprotein (LDL) is one of the five major groups of lipoproteins, which, in order of molecular size, largest to smallest, are chylomicrons, very low-density lipoprotein (VLDL), intermediate-density lipoprotein (IDL), LDL, and high-density lipoprotein (HDL). Lipoprotein molecules enable the transportation of lipids (fats), such as cholesterol, phospholipids, and triglycerides, in the extracellular fluid including the bloodstream. Studies have shown that higher levels of type-B LDL particles (as opposed to type-A LDL particles) are associated with health problems, including cardiovascular disease [9].

Although the nickname is overly simplistic and thus misleading, LDL molecules are often informally called bad cholesterol because they can transport their content of many fat molecules into artery walls, attract macrophages, and thus drive atherosclerosis. In contrast, HDL molecules are frequently referred to as good cholesterol or healthy cholesterol, because they can remove fat molecules from macrophages in the wall of arteries [10].

Alanine transaminase (ALT (SGPT) is solely found in the liver. It is a biomarker of liver injury in a patient with some degree of intact liver function. Its plasma level is raised in liver damage [11].

This work was therefore, designed to evaluate the effect of liquid extract of pear leaf used in the traditional treatment of hypertension on the plasma levels of aminotransferases, cholesterol and total bile acids

\section{Materials and Methods}

\section{Materials:}

\section{a. Blood Sample}

Five (5) milliliter of blood were collected from each of the patients before the administration of the pear leaf liquid extract for the baseline plasma Alanine transaminase, Aspartate transaminase, Total Bile Acids, LDL and Total Cholesterol. Another blood sample was collected from each of the patients after an appreciable decrease in blood pressure.

\section{b. Preparation of the raw Liquid extract of pear avocado}

The leaves of the pear avocado fruit are plucked and washed in water. The water is drained and the leaves are crushed or squished for the extraction of the liquid content into a container. A plastic cup with a capacity of $60 \mathrm{ml}$ was dedicated by the healers for the measurement of the extract to be administered into the patients. The liquid extract is freshly prepared on daily bases prior to administration. The raw liquid content of the leaf is extracted without the addition of water and also administered undiluted.

\section{c. Reagents}

They include: Randox test kit of Randox for TBA estimation and Roche Cobas C111 compatible reagent kit was used for the estimation ALT, AST, Total and LDL Cholesterol.

\section{Study area}

The study was carried out in ATISBO, Saki-East and Saki-West Local government areas of Oke-Ogun - the Northern part of Oyo state-Nigeria. The three local governments constitute the former Ifedapo Local government area of Oyo state. The three local governments share border with Kwara state-Nigeria, Ogun state-Nigeria and the Republic of Benin.

\section{Study design}

Experimental research design.

\section{Study population}

a. Fifty (50) newly diagnosed hypertensive patients with an abnormal increase in plasma cholesterol and blood pressure aged 45 and above that have not been treated with any hypertensive medication but have decided to be treated traditionally using pear leaf extract were recruited from the traditional homes in ATISBO, Saki-East and Saki-West Local government areas of Oke-Ogun - the Northern part of Oyo state-Nigeria. None of the subject was jaundiced as at the time of sample collection.

\section{Sample size}

Fifty (50) out of the sixty eight (68) hypertensive patients (Female: $\mathrm{n}=25$; Male: $\mathrm{n}=25$ ) that visited the traditional healers between September, 2013 and February, 2014 for treatment that volunteered themselves for this study were recruited based on the inclusion and the exclusion criteria.

\section{Case selection procedure/s \\ Inclusion criteria}

Anicteric newly diagnosed hypertensive patients with abnormal increase in plasma cholesterol and blood pressure aged 45 and above that have not been treated with any hypertensive medication were recruited.

\section{Exclusion criteria}

1. Hypertensive patients that have been treated or being treated with antihypertensive drugs were not recruited for the study. 
2. Anicteric newly diagnosed hypertensive patients with normal plasma cholesterol aged 45 and above that have not been treated with any hypertensive medication were not included in the study.

3. Icteric newly diagnosed hypertensive patients with abnormal increase in plasma cholesterol and blood pressure aged 45 and above that have not been treated with any hypertensive medication were not included for the study.

\section{Methods:}

a. Based on the information obtained from the fifteen traditional homes visited in the three local governments about $60 \mathrm{ml}$ of the raw undiluted liquid extract of the pear leaves is administered to the patients on daily bases until there is an appreciable decrease in the blood pressure to an acceptable range.

b. Estimation of plasma Aspartate transaminase, Alanine Transaminase, Total cholesterol and LDL Cholesterol were carried out using Cobas C111 auto-chemistry analyzer using the reagent kit of Roche.

c. Estimation of Total Bile Acids was carried out on the plasma samples of the subjects using Randox reagent kit. The manufacturer's instruction was strictly followed.

Principle: Two reactions are combined in this kinetic enzyme cycling method. In the first reaction bile acids are oxidised by 3- $\alpha$ hydroxysteroid dehydrogenase with the subsequent reduction of Thio-NAD to Thio-NADH. In the second reaction the oxidised bile acids are reduced by the same enzyme with the subsequent oxidation of NADH to NAD. The rate of formation of Thio-NADH is determined by measuring the specific absorbance change at $405 \mathrm{~nm}$.

(Abreviations: NADH, NAD, Thio-NADH, Thio-NAD)

\section{d. Ethical Consideration}

The proposal was reviewed and approved by the Research and Ethical Committee of Baptist Medical Centre, Saki-Oyo state- Nigeria before the commencement of the work. This is to protect the interest of patients to ensure that the patients and the community are not harmed in any form by the procedure

\section{e. Statistical Analysis}

the values of the biochemical parameters obtained in the patients before and after treatment with the raw liquid extract were subjected to statistical analysis to determine the mean values, standard deviation and students' ' $\mathrm{t}$ ' test, for $\mathrm{t}$ value, $\mathrm{p}$ value and level of significant at $0.01(99 \%)$ using online Student T-Test Calculator for 2 Independent Means on line at. http://www.socscistatistics.com/tests/studentttest

\section{Result}

The results obtained considering tables 1 and 2 showed a significantly higher mean value of plasma Total and LDL Cholesterol in the subjects before the administration of the liquid extract of avocado pear leaf than the values obtained post-administration with $\mathrm{p}<0.01$. However there was a significantly lower mean value of plasma ALT and AST in the subjects before the administration of the liquid extract of avocado pear leaf than the values obtained post-administration with $\mathrm{p}<0.01$.(Table 1 and 2 ). There was a non-statistically higher plasma value of Total Bile Acids in the subjects before the administration of the raw liquid extract of avocado pear leaf than the value obtained in the subjects post-administration with $\mathrm{p}>0.01$ (Table 1 and 2).

Table 1. Result of the Mean and the Standard deviation of the plasma values of ALT, TBA, AST, Total and LDL Cholesterol in the subjects before and after treatment with the Liquid extract of pear avocado leaf

\begin{tabular}{|c|c|c|}
\hline \multirow{2}{*}{ Measurements } & \multicolumn{2}{|c|}{ Mean Values with SD } \\
\cline { 2 - 3 } $\mathbf{A L T}$ & Before treatment & After treatment \\
\hline $\mathbf{U} / \mathbf{L}$ & $20 \pm 1.0$ & $48 \pm 2.1$ \\
\hline TBA $\boldsymbol{\mu m o l} / \mathbf{L}$ & $8.0 \pm 1.5$ & $5.0 \pm 1.8$ \\
\hline $\begin{array}{c}\text { AST } \\
\text { U/L }\end{array}$ & $14.0 \pm 2.4$ & $42 \pm 1.2$ \\
\hline $\begin{array}{c}\text { Total Cholesterol } \\
\mathbf{m g} / \mathbf{d l}\end{array}$ & $281 \pm 10.0$ & $180 \pm 2.1$ \\
\hline $\begin{array}{c}\text { LDLCholesterol } \\
\mathbf{m g} / \mathbf{d l}\end{array}$ & $160 \pm 5.0$ & $96 \pm 2.2$ \\
\hline
\end{tabular}

Table 2. Results of the students' ' $t$ ' test, probability(' $p$ ') and the level of significance of the differences in plasma values of ALT, TBA, AST and Cholesterol in the subjects before and after treatment

\begin{tabular}{|c|c|c|c|}
\hline & 't' value & 'p' value & Comment \\
\hline ALT & 13.8 & 0.005 & $\mathrm{P}<0.01^{\mathrm{S}}$ \\
\hline TBA & 1.06 & 0.4 & $\mathrm{p}>0.01^{\mathrm{NS}}$ \\
\hline AST & 12.5 & 0.006 & $\mathrm{P}<0.01^{\mathrm{S}}$ \\
\hline Total Cholesterol & 10.95 & 0.008 & $\mathrm{P}<0.01^{\mathrm{S}}$ \\
\hline LDL Cholesterol & 11.8 & 0.007 & $\mathrm{P}<0.01^{\mathrm{S}}$ \\
\hline
\end{tabular}

S-Significant, NS- Not significant, ' $\mathbf{t}$ '=student ' $\mathrm{t}$ ' test, ' $\mathbf{p}$ ' = probality value

\section{Discussion}

Hypertensive patients with abnormal increase in plasma total and LDL cholesterol levels were studied in this work because of the contribution of the lipid to the cause of heart disease. This could be supported by the report of CDC [12] that high blood pressure and cholesterol are the two main reasons that could be associated with the deadly but preventable heart disease or stroke. Nearly 2 out of 3 adults with high cholesterol and about half of adults with high blood pressure do not have their condition yet under control. Clearly, other steps are needed to gain control of these health risks [12].

High cholesterol accelerates the progression of atherosclerosis, which is thought to contribute significantly to hypertension [12]. Atherosclerosis of renal artery leading to renovascular diseases (arterial stenosis) which ended up with less blood flow to the kidney and subsequent triggering of renin-angiotensin-aldosterone system that involves a variety of hormones to increase blood pressure.

The increase in blood pressure necessary to return kidney 
blood flow to normal is often much greater than normal blood pressure; consequently these patients develop hypertension. Which doubles coronary heart disease (CHD) risk. Treating hypertension only reduces CHD risk $\approx 25 \%$ while Treating hypercholesterolemia in hypertensive patients reduces residual CHD risk $>35 \%$ [12].

The results obtained showed a lowering effect of the extract on plasma Total and LDL Cholesterol in the hypertensive subjects. This could be associated with the relevance of the extract in the treatment of hypertension due to arteriosclerosis caused by abnormal increase in Total and LDL cholesterol. This biochemical decrease in the plasma level of Total and LDL cholesterol in the hypertensive patients after the administration of the pear leaf extract could be due to the effect of the phytochemical constituents of the leaf which include: Tannins, Saponins, Cardiac glycosides, Alkaloids and [5]. These chemical substances are also found in Buccholzia coriacea seeds reported by Omolekan and Olaiya [13] responsible for the reduction in plasma LDL cholesterol in hypercholesterolemic rats treated with the alcoholic extract of the seed and that the extract is potent in reducing the formation of plaques in the coronary arteries and also the generation of atherosclerosis.

The health benefits of saponin include cholesterol lowering and anticancer properties [16]. Alkaloids have been found to possess analgesic, antimalarial, antibacterial, antihypertensive [17] properties. Pigments like tannins and flavonoids may have been responsible for this cholesterol lowering action. Phytochemicals like tannins, saponins and flavonoids are constituents of plant extracts such as avocado pear that have lipid lowering. These phytochemicals also prevent the oxidation of LDL, preventing it from being atherogenic $[18,19]$.

When too much LDL (bad) cholesterol circulates in the blood, it can slowly build up in the inner walls of the arteries that feed the heart and brain. Together with other substances, it can form atherosclerosis characterized by a thick, hard deposit that can narrow the arteries and make them less flexible, and if a clot forms and blocks a narrowed artery, heart attack or stroke can result. The formation of plaque could be affected by the phytochemical constituents of the extract thereby preventing atherosclerosis and consequently preventing hypertension [2].

In this study a significant increase in plasma ALT and AST in the subject after the administration of the liquid extract indicates that it seems to have a toxic effect on the liver that could cause liver damage which is slightly comparable with the report of Friday et al., [14] that reported a non-statistical increase in plasma ALT, AST in albino rat after the administration aqueous extract of the seed of Avocado and African pear seeds.

There was a non-statistically higher plasma value of Total Bile Acids in the subjects before the administration of the raw liquid extract of avocado pear leaf compared with the values obtained in the subjects after the administration of the extract. This could be attributed to the metabolic relationship between the Cholesterol and Total Bile Acids. In this study there is a decrease in the plasma cholesterol level following the administration of the extract which could also affect the metabolism of bile acids [9].

\section{Conclusions}

The findings of this study revealed a significant alteration in the plasma level of ALT, AST, LDL and Total Cholesterol in the subjects after the administration of the liquid extract. A significant increase in plasma ALT and AST in the subject after the administration of the liquid extract indicates that the extract seems to have a toxic effect on the liver that could cause liver damage. Consequently, evaluation of these biochemical parameters in hypertensive patients undergoing traditional therapy using the extract is therefore recommended.

\section{REFERENCES}

[1] Anslem, A. Nature Power. Christian Approach to Herbal Medicine. Don Bosco Training Centre, School of PTP, Printing and Finishing, Akure. 2006: 206p.

[2] Okoli, R. I., Aigbe, O., Ohaju - Obodo, J. O, and Mensah, J. $\mathrm{K}$. Medicinal herbs usedfor managing some common ailments among Esan people of Edo state, Nigeria. Pakistan Journal of Nutrition 2007: 6(5): 490 - 496,

[3] Ayitey - Smith, E. Prospect and Scope of Plant Medicine in Health Care. Ghana University Press, Accra. 1989: 29pp,.

[4] Odugbemi, T. T. Outline and Pictures of Medicinal Plants from Nigeria. University of Lagos Press, Lagos, Nigeria, $2006: 283 p p$.

[5] Mensah J.K., Okoli R I., Turay A. A. and Ogie-Odia E.A. Phytochemical Analysis of Medicinal Plants Used for the Management of Hypertension by Esan people of Edo State, Nigeria. Ethnobotanical Leaflets2009: 13: 1273-87.

[6] Sadava D, Hillis DM, Heller HC, Berenbaum MR. Life: The Science of Biology 9th Edition. San Francisco: Freeman. 2011: pp. 105-114. ISBN 1-4292-4646-4.

[7] John S, Sorokin AV, Thompson PD. "Phytosterols and vascular disease". Curr.Opin.Lipidol. 2007: 18 (1): 35-40.. doi:10.1097/MOL.0b013e328011e9e3.PMID 17218830.

[8] Ohvo-Rekilä H, Ramstedt B, Leppimäki P, Slotte JP. "Cholesterol interactions with phospholipids in membranes". Prog. Lipid Res. 2007: 41 (1): 66-97. 2002. doi:10.1016/S0163-7827(01)00020-0. PMID 11694269.

[9] Dashty M, Motazacker MM, Levels J, de Vries M, Mahmoudi M, Peppelenbosch MP, Rezaee F. "Proteome of human plasma very low-density lipoprotein and low-density lipoprotein exhibits a link with coagulation and lipid metabolism.". ThrombHaemost. 2014: 23 (111): 518-530.. doi:10.1160/TH13-02-0178. PMID 24500811

[10] Dashti M, Kulik W, Hoek F, Veerman EC, Peppelenbosch MP, Rezaee F. "A phospholipidomic analysis of all defined human plasma lipoproteins.". Sci Rep. 2011: 1 (139).. doi:10.1038/srep00139. PMID 22355656. 
[11] McClatchey, Kenneth D. Clinical laboratory medicine. Lippincott Williams \& Wilkins2002:pp. 288-.ISBN 978-0-683-30751-1.

[12] Centers for Disease Control and Prevention (CDC) High Blood Pressure and Cholesterol Out of Control February 2011. http://www.cdc.gov/Vitalsigns/CardiovascularDisease/

[13] T. O. Omolekan1 and C. O. O. Olaiya. Anti - Atherogenic Activity of Ethanol Extract of Buccholziacoriacea Seeds on Hypercholesterolemic Rats. International Journal of Biochemistry Research \& Review2013: 3(3): 190-199, SCIENCEDOMAIN international www.sciencedomain.org

[14] Friday O.U, Amadike C.U, and Kingsley C.N. Effect of aqueous extract of Pear seeds on some biochemical parameters in albino rats. A comparative study of Persea Americana (Avocado pear) and Dacryodesedulis (African pear). Journal of Reasearch in Biochemistry. 2013: 2(1): 110 -115 ,

[15] Brent M. Egan, MD; Jiexiang Li, PhD; SuparnaQanungo,
PhD; Tamara E. Wolfman, MD. Blood Pressure and Cholesterol Control in Hypertensive Hypercholesterolemic Patients. National Health and Nutrition Examination Surveys 1988-2010.

[16] Kim, S.W, S.K. Park, S.I. Kang, H.C. Kang, H.J. Oh, C.Y. Bae and D.H. Bae, Hypocholesterolemic property of Yucca schidigera and Quillaja saponaria extracts in human body. 2003:Arch Pharm. Res., 26: 1042-1046

[17] Dangi, S.Y., C.I. Jolly and S. Narayanan, Antihypertensive activity of the total alkaloids from the leaves of Moringa oleifera. Pharm. Biol., 2002:40(2): 144-148.

[18] Adeneye AA, Adeneke TI, Adeneye AK.Hypoglycemic and hypolipidemic effects of the aqueous leaves extracts of Clerodendrum capitatum in wistar rats. J. Ethanopharmacol., 2008: 116(1): 7-10

[19] Ram A, Laura P, Gupta R, Kumar P, Sharma VN. Hypocholesterolaemic effects of Terminalia arguna tree bark. J. Ethanopharmacol., 1997 :55(3): 165-169. 